\title{
Calorimetric neutrino expectations from bright blazar flares
}

\author{
Michael Kreter ${ }^{* a}$, Matthias Kadler ${ }^{b}$, Felicia Krauß ${ }^{c}$, Sara Buson ${ }^{b}$, Roopesh Ojha ${ }^{d}$ for \\ the Fermi/LAT Collaboration, Karl Mannheim ${ }^{b}$, Jörn Wilms ${ }^{e}$ and Markus Böttcher ${ }^{a}$ \\ ${ }^{a}$ Centre for Space Research, North-West University, Private Bag X6001, Potchefstroom 2520, \\ South Africa \\ ${ }^{b}$ Lehrstuhl für Astronomie, Universität Würzburg, Emil-Fischer-Straße 31, 97074 Würzburg, \\ Germany \\ ${ }^{c}$ GRAPPA \& Anton Pannekoek Institute for Astronomy, University of Amsterdam, Science \\ Park 904, 1098 XH Amsterdam, The Netherlands \\ ${ }^{d}$ NASA Goddard Space Flight Center, Greenbelt, MD 20771, USA \\ ${ }^{e}$ Dr. Remeis Sternwarte \& ECAP, Universität Erlangen-Nürnberg, Sternwartstraße 7, 96049 \\ Bamberg, Germany \\ michaelakreter.org
}

\begin{abstract}
Blazar jets are extreme environments, in which relativistic proton interactions with an ultraviolet photon field could give rise to photopion production. High-confidence associations of individual high-energy neutrinos with blazar flares could be achieved via spatially and temporally coincident detections. In 2017, the track-like, extremely high-energy neutrino event IC 170922A was found to coincide with increased $\gamma$-ray emission from the blazar TXS 0506+056, leading to the identification of the most promising neutrino source candidate so far. We test the chance coincidence of such events by calculating the expected number of neutrinos that can be detected by IceCube, based on a broadband parametrization of bright short-term blazar flares that were observed in the past 8 years by Fermi/LAT. We find that the integrated keV-to-GeV fluence of most individual blazar flares is far too small to yield a substantial Poisson probability for the detection of one or more neutrinos with IceCube. In contrast to such short-term flares that usually last only a few days or less, TXS0506+056 did show a major outburst that lasted several months, giving rise to a much higher fluence than most short blazar flares. We show, based on the calorimetric argumentation presented in this work, that the association of the IC 170922A neutrino with TXS 0506+056 is energetically plausible at a significance level of about 3.5 sigma. We further discuss strategies to search for more significant associations in future data unblindings of IceCube and KM3NeT.
\end{abstract}

36th International Cosmic Ray Conference -ICRC2019-

July 24th - August 1st, 2019

Madison, WI, U.S.A.

${ }^{*}$ Speaker. 


\section{Introduction}

Jets from Active Galactic Nuclei (AGN) are promising candidates for the detected high-energy neutrino flux $[1,2]$. Even if the contribution from blazars to the total high-energy neutrino flux measured by IceCube is small $[3,4,5]$, we have shown in our previous work that the contribution from individual blazar flares is not negligible [6, 7]. While general neutrino point-source searches $[8,9,10,11]$ focus on a simultaneous correlation to the observed blazar $\gamma$-ray flux, we have shown in [12] that the total high-energy fluence is a much better proxy for the expected number of observable neutrino events. On 22 September 2017, IceCube detected a $\sim 290 \mathrm{TeV}$ neutrino-induced, track-like event (called IC 170922A) from a direction spatially and temporally consistent with enhanced $\gamma$-ray activity from the blazar TXS 0506+056. Recent models developed in this context indicate that the neutrino emission from $p \gamma$ interactions can be best described by a lepto-hadronic model with a weak hadronic component $[13,14,15]$. Such models predict a correlation between the observed X-ray/TeV emission and the measured neutrino event. Other models [16] suggest a stationary soft-X-ray photon target field in a photo-hadronic neutrino production scenario, which leads to a decoupling of the observed $\gamma$-ray emission and the measured neutrino flux.

In this work, we present a (model-independent) calorimetric approach to estimate the number of predicted high-energy neutrino events an individual blazar flare would be able to produce. We compute the expected neutrino excess rates of bright blazar flares by performing a systematic search for flaring intervals on different time scales. We also study the $\gamma$-ray variability and neutrino prediction of TXS 0505+056 and show that a calorimetric association between IC 170922A and a long-term outburst period of several months seems to be reasonable.

\section{Method}

\subsection{Calorimetric Neutrino Expectation}

While neutrinos are created exclusively in hadronic interactions, high-energy $\gamma$-rays can originate from both leptonic and hadronic interactions. We use the calorimetric approach following [6] and [7] in order to estimate the maximum amount of high-energy neutrino events, a particular flare would be able to produce in a $p \gamma$-interaction. This leads to a balance between the integrated $\gamma$-ray energy flux $\Phi_{\gamma}$ and the integrated neutrino energy flux $\Phi_{v}$, following [12]:

$$
\Phi_{v} \approx \Phi_{\gamma}
$$

In this maximum case, Monte Carlo simulations showed that this is a reasonable approximation for blazars [17]. The maximum number of neutrino events a flare can create is given by:

$$
N_{v}^{\max }=\mathrm{A}_{\mathrm{Eff}} \times\left(\frac{\Phi_{v}}{E_{v}}\right) \cdot \Delta t
$$

$A_{\text {Eff }}$ represents the energy-dependent IceCube effective area taken at a neutrino energy of $E_{v}=$ $2 \mathrm{PeV}, \Phi_{v}$ the maximum integrated neutrino energy flux and $\Delta t$ the duration of the selected flare, assuming the emission of all $\gamma$-rays and neutrinos to happen during this time interval. To get a 
more realistic estimate of how many neutrino events one might indeed expect to be detectable in the IceCube detector, one can compute the predicted neutrino number according to:

$$
N_{v}^{\text {pred }}=f \times N_{v}^{\max } .
$$

Following [7], the scaling factor ${ }^{1} f$ can be factorized into a flavor factor $f_{I}$, a factor accounting for the different classes of blazars $f_{I I}$, and a spectrum factor $f_{I I I}$ :

$$
f=f_{I} \times f_{I I} \times f_{I I I}=0.025
$$

To account for the maximum integrated neutrino energy flux $\Phi_{v}$ a given flare can generate, we integrate over its entire high-energy emission of the blazar spectral energy distribution (SED) in an energy range of $10 \mathrm{keV}$ to $20 \mathrm{GeV}$. This energy range covers the peak of the high-energy component and ensures that the total X-ray to $\gamma$-ray emission is considered in our integrated flux estimate. Simultaneous Swift/XRT and Fermi/LAT observations of each flare are used to build SEDs, whose high-energy hump can be parameterized by a log-parabola model.

\subsection{Flare Identification}

We used daily binned Fermi/LAT $\gamma$-ray light curves of a sample of 150 bright blazars monitored daily by the $\mathrm{LAT}^{2}$, to identify periods of enhanced activity according to:

$$
\Delta_{\text {flare }}=\left[\left(\text { Flux }-3 \times \text { Flux }_{\text {err }}\right) \geq 3 \mathrm{G}\right] \times \mathrm{A}_{\mathrm{Eff}}
$$

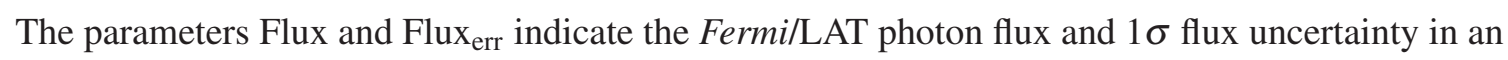
energy range of $100 \mathrm{MeV}-300 \mathrm{GeV}$. G corresponds to a (source dependent) non-flaring quiescent ground level state. The quantity $\Delta_{\text {flare }}$ therefore defines a threshold selection criteria for flares of a considered blazar light curve. The ground level $\mathrm{G}$ is derived by creating a histogram of Fermi/LAT fluxes and fitting the distribution with a Gaussian function. From the mean of the Gaussian fit we derive the ground level flux of the studied blazar. The tail of this flux distribution histogram corresponds to flux values within flaring states. The IceCube effective area $A_{E f f}$ ensures a weighting of identified flares according to the IceCube sensitivity. This flare identification is optimized to select bright blazar flares above a certain (source specific) threshold. Short flares on daily to weekly time scales are favored, as no additional assumption about the flaring behavior is required. Longer outburst periods, which are interrupted by non-detections are therefore split into several individual flares, as in this selection a flare corresponds to an uninterrupted period of activity.

\section{Potential high-energy Neutrino-Blazar Associations}

Blazars show a complex flaring behavior in the high-energy regime. Here, we present the neutrino predictions from a systematic search for bright blazar flares with durations of days to weeks. We also study the long-term flaring behavior of TXS 0506+056 with Fermi/LAT and calculate the neutrino expectation rates for different flare durations.

\footnotetext{
${ }^{1}$ Here we consider an empirical scaling in a purely hadronic scenario for Flat-Spectrum Radio Quasars. The actual neutrino prediction is expected to be even lower by using a more realistic lepto-hadronic model as shown in [12]

${ }^{2}$ All these light curves are publicly available at http://fermi.gsfc.nasa.gov/ssc/data/access/ lat/msl_lc/
} 


\subsection{Bright $\gamma$-ray Blazar Flares}

Table 1 lists the results of the neutrino expectation for a subsample of 50 flares identified in Sect. 2.2. Flares which show the highest time averaged integrated $\gamma$-ray flux

$$
\left\langle\Phi_{\gamma}\right\rangle=\frac{1}{\Delta t} \int_{0}^{\Delta t} \Phi_{\gamma}(t) d t
$$

have been selected. In order to compare the neutrino expectation of flares with various durations, it is necessary to normalize the neutrino predictions of individual flares according to their duration. The neutrino rate per flare is given by:

$$
\left\langle\dot{N}_{v}^{\text {flare }}\right\rangle=\frac{\mathrm{N}_{v}^{\text {pred }}}{\Delta t}
$$

While longer flares lead to higher absolute neutrino numbers (see Eq. 2.2), the chance coincidence for a correlated detection of a high-energy neutrino and a $\gamma$-ray flare is expected to be higher for shorter flare durations. The neutrino rate therefore allows us to compare the neutrino prediction for flares with various time span. While the majority of flares seem to have similar neutrino rates, unaffected by their duration, 3C 279 underwent a massive flare in 2015 [18] which clearly dominates the sample by producing the highest time averaged integrated $\gamma$-ray flux as well as the highest neutrino rate. The two blazars 3C 279 and PKS 1510-089 alone account for a majority of 42 flares, while the 50 highest-ranked flares are produced by a group of only seven different sources: 3C 279 (10 flares), PKS 1510-089 (32 flares), PKS 0402-362 (1 flare), CTA 102 (1 flare), 3C 454.3 (1 flare), PKS 1424-418 (3 flares), and PKS 1329-049 (2 flares). Flare 38 clearly provides the highest absolute neutrino prediction due to its brightness and duration of multiple months. Comparing this flare to the "BigBird" event studied by [7], the chance coincidence for a correlated high-energy neutrino detection is expected to be small. Studying the neutrino rate, we see that in order to maximize the chance coincidence for an astrophysical neutrino detection, flares need to have a high integrated $\gamma$-ray energy flux on a relatively short time scale.

\subsection{TXS 0506+056: A Promising Neutrino Point Source Candidate}

We performed a long-term variability analysis in a time interval of nine years ranging from the start of the Fermi mission in August 2008 until the arrival of IC 170922A in September 2017. Figure 1 shows the long-term Fermi/LAT $\gamma$-ray light curve in an energy range of $100 \mathrm{MeV}$ to $300 \mathrm{GeV}$ using monthly time bins. It is worth mentioning, that compared to the bright blazar flares typically studied by Fermi/LAT, such an outburst is not unusual for a BL Lac object and even brighter flares have been observed from various BL Lacs. [19, 20].

While many neutrino point-source studies (e.g. $[10,11])$ focus on a neutrino $\gamma$-ray correlations in a narrow time windows around the arrival time of the neutrino event, no short-term flaring period according to Sect.2.2 is identified in September 2017. In the following, we are computing the calorimetric neutrino expectation for a typical short-term period as well as a long-term period of several months. 


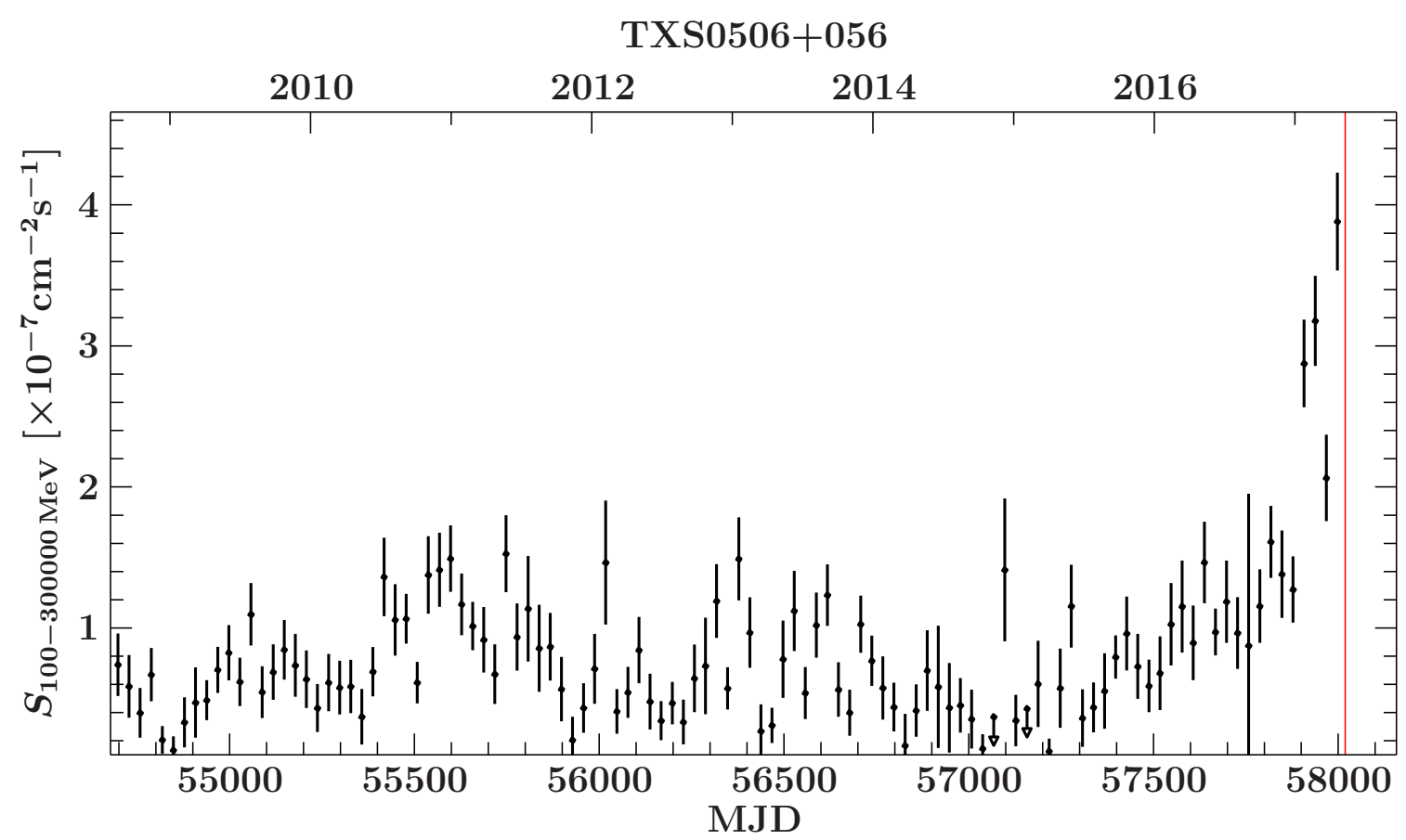

Figure 1: Fermi/LAT $\gamma$-ray light curve of TXS $0506+056$ in an energy range of $100 \mathrm{MeV}-300 \mathrm{GeV}$ and a time range of 54682.65 MJD - 58027 MJD using monthly binning. The arrival time of IC $170922 \mathrm{~A}$ is indicated by a red line.

\subsubsection{Neutrino Predictions on Various Time Scales}

A time interval of three days ranging from 58017 MJD - 58020MJD is defined around the arrival time of the neutrino event in order to compare the neutrino prediction of this short time range to follow up studies typically performed [9, 21, 22]. Following our calorimetric argumentation, we estimate the maximum, mono-energetic neutrino output detectable by IceCube to be:

$$
N_{v}^{\text {pred }} \approx 9 \times 10^{-5}
$$

This number is small, even compared to the results from other flares found in Table 1 with similar duration. This expectation is due to the fainter nature of this source at X-ray and $\mathrm{GeV}$ energies, whose time averaged integrated $\gamma$-ray flux is reduced by about two orders of magnitude. From a calorimetric point of view and contradictory to the majority of follow up studies, it seems unlikely to associate the event IC 170922A with a short-term flare period of the blazar TXS 0506+056.

The outburst period at $\mathrm{MeV}$ to $\mathrm{GeV}$ energies, during which IC 170922A occurred started in April 2016, as seen in Fig. 1. As the number of neutrino events (Equ. 2.2) scales linearly with the duration of the flare, we expect more neutrinos to be produced during a long-term outburst. For the interpretation it is important to remember that a considerable number of other blazars have produced similar outbursts, which did not coincide with the detection of a high-energy neutrino due to the small Poisson probability. Thus, it is not surprising that the neutrino prediction we derived is well below unity. Similar values from short-term blazar flares can only be achieved by producing a much higher averaged flux on relatively short time scales. Thus, based on the used calorimetric approach, 
an association of the high-energy neutrino event IC 170922A with the blazar TXS 0506+056 seems calorimetrically more reasonable.

\section{Conclusion and Outlook}

The calorimetric estimate presented in this work is based on an equal balance of neutrinos and $\gamma$-rays, which clearly overestimates the number of predicted neutrino events. Even by using these certainly over-optimistic assumptions, we showed that the absolute neutrino expectation for most short-term blazar flares is too small to be considered for a dedicated correlation analysis. However, for the most spectacular short-term blazar flares, we derived neutrino numbers comparable to the expectations from long-term outbursts, so that the possibility for associated neutrino detections, seams reasonable ${ }^{3}$.

We studied the flaring behavior of TXS 0506+056 with Fermi/LAT and showed that from a calorimetric point of view an association between IC 170922A and a short-term flaring period around the neutrino arrival time is unlikely. This result is mainly driven by the faint integrated $\gamma$-ray energy flux of this source in comparison to flares studied in Sect. 3.1. Since 2016, this blazar underwent an enhanced phase of activity. A correlation to this long-term outburst period seems calorimetrically more realistic. Independently, in a time range between September 2014 and March 2015 the IceCube Collaboration [24] found an excess of high-energy neutrino events, with respect to atmospheric background, providing a $\sim 3.5 \sigma$ evidence for neutrino emission from the direction of TXS 0506+056. This further suggests that blazars are identifiable sources of the extragalactic high-energy neutrino flux, and especially the source TXS $0506+056$ to be a promising candidate for the first high-energy neutrino point source.

In order to put these two studies into context it is important to mention that the result we derived assumes the event IC 170922A to be of astrophysical origin, which is highly uncertain. ${ }^{4}$ In a more realistic lepto-hadronic scenario, however, blazars do show a varying hadronic contribution to the observed integrated $\gamma$-ray energy flux, which will further reduce the number of expected neutrino events per flare [12]. Future neutrino point-source studies, beside searching for a correlation of high-energy neutrinos and $\gamma$-ray flares with various durations, should in addition consider the hadronic emission to peak more towards the X-ray or optical regime. The All-sky Medium Energy Gamma-ray Observatory (AMEGO; [25]) will survey the entire sky in an energy range of $200 \mathrm{keV}$ to $10 \mathrm{GeV}$ and provide crucial information for the understanding of the emission mechanisms in blazar jets. [26].

\footnotetext{
${ }^{3}$ Taking into account that the Poisson probability is still on the order of only a few per cent.

${ }^{4}$ https://gcn.gsfc.nasa.gov/notices_amon/50579430_130033.amon
} 
Table 1: Neutrino expectation for the 50 best-ranked flares, selected according to their neutrino rate $\left\langle N_{v}^{\text {flare }}\right\rangle$ (see Eq. 3.1). $t_{\min }$ and $t_{\max }$ indicate the start and stop time of a flare in Modified Julian Day (MJD). Flares have been sorted according to their integrated $\gamma$-ray energy flux $\left\langle\Phi_{\gamma}\right\rangle$. For the full table, please refer to [23].

\begin{tabular}{|c|c|c|c|c|c|c|c|c|}
\hline Source & $\begin{array}{c}\text { Flare } \\
\text { Number }\end{array}$ & $\left\langle\Phi_{\gamma}\right\rangle$ & $\begin{array}{c}\mathbf{t}_{\min } \\
{[\mathrm{MJD}]}\end{array}$ & $\begin{array}{c}t_{\max } \\
{[\mathrm{MJD}]}\end{array}$ & $\mathbf{N}_{v}^{\max }$ & $\mathbf{N}_{v}^{\text {pred }} \times 10^{-2}$ & $\begin{array}{c}\text { Duration } \\
\text { [Days] }\end{array}$ & $\left\langle N_{v}^{\text {flare }}\right\rangle \times 10^{-3}$ \\
\hline 3C 279 & 1 & 260238 & 57186 & 57192 & 0.797 & 1.99 & 6 & 3.32 \\
\hline PKS $1510-089$ & 2 & 192902 & 55849 & 55854 & 0.306 & 0.764 & 5 & 1.53 \\
\hline PKS $1510-089$ & 3 & 151569 & 55866 & 55877 & 0.586 & 1.46 & 11 & 1.33 \\
\hline PKS $1510-089$ & 4 & 151262 & 55856 & 55857 & 0.0405 & 0.101 & 1 & 1.01 \\
\hline 3C 279 & 5 & 138636 & 56717 & 56718 & 0.0272 & 0.0681 & 1 & 0.681 \\
\hline $3 \mathrm{C} 279$ & 6 & 128078 & 56749 & 56754 & 0.214 & 0.535 & 5 & 1.07 \\
\hline PKS $1510-089$ & 7 & 125857 & 57241 & 57251 & 0.393 & 0.982 & 10 & 0.982 \\
\hline 3C 279 & 8 & 119379 & 56866 & 56868 & 0.0993 & 0.248 & 2 & 1.24 \\
\hline PKS $1510-089$ & 9 & 119033 & 56553 & 56557 & 0.159 & 0.398 & 4 & 0.995 \\
\hline PKS 1510-089 & 10 & 116956. & 55766 & 55768 & 0.0605 & 0.151 & 2 & 0.757 \\
\hline PKS $1510-089$ & 11 & 114301 & 57098 & 57099 & 0.0422 & 0.105 & 1 & 1.05 \\
\hline PKS $1510-089$ & 12 & 111097 & 56563 & 56565 & 0.0652 & 0.163 & 2 & 0.815 \\
\hline PKS $1510-089$ & 13 & 110472 & 55843 & 55844 & 0.0305 & 0.0763 & 1 & 0.763 \\
\hline PKS $1510-089$ & 14 & 109350 & 57148 & 57149 & 0.0310 & 0.0775 & 1 & 0.775 \\
\hline PKS $1510-089$ & 15 & 104003 & 56542 & 56543 & 0.0283 & 0.0707 & 1 & 0.707 \\
\hline PKS $1510-089$ & 16 & 102145 & 55742 & 55746 & 0.0889 & 0.222 & 4 & 0.555 \\
\hline PKS $1510-089$ & 17 & 93862 & 56610 & 56611 & 0.0223 & 0.0560 & 1 & 0.560 \\
\hline PKS $1510-089$ & 18 & 93711 & 55738 & 55740 & 0.0480 & 0.120 & 2 & 0.600 \\
\hline PKS $1510-089$ & 19 & 91983. & 55954 & 55994 & 1.61 & 4.03 & 40 & 1.01 \\
\hline PKS 1510-089 & 20 & 91625 & 55997 & 56006 & 0.403 & 1.01 & 9 & 1.12 \\
\hline : & : & : & 8 & : & 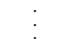 & : & : & : \\
\hline $3 \mathrm{C} 454.3$ & 38 & 74620 & 55408 & 55648 & 11.71 & 29.28 & 240 & 1.22 \\
\hline & & : & : & : & : & : & : & \\
\hline PKS $1510-089$ & 50 & 66603 & 56567 & 56569 & 0.0393 & 0.0983 & $\dot{2}$ & 0.491 \\
\hline
\end{tabular}

\section{References}

[1] K. Mannheim, The proton blazar, A\&A 269 (1993) 67-76 [arXiv:astro-ph/9302006]

[2] K. Mannheim, High-energy neutrinos from extragalactic jets, Astroparticle Physics 3 (1995) 295-302

[3] Turley et. al., Search for Blazar Flux-correlated TeV Neutrinos in IceCube 40-string Data, ApJ 833 (2016) 117 [arXiv:1608.08983]

[4] Aartsen et. al., Constraints on Ultrahigh-Energy Cosmic-Ray Sources from a Search for Neutrinos above 10 PeV with IceCube, Phys. Rev. Lett. 117 (2016) 241101 [arXiv:1607.05886]

[5] Murase et. all, Constraining high-energy cosmic neutrino sources: Implications and prospects, Phys. Rev. D 94 (2016) 103006 [arXiv:1607.01601]

[6] Krauß et. all, TANAMI blazars in the IceCube PeV-neutrino fields, A\&A 566 (2014) L7 [arXiv:1406.0645]

[7] Kadler et. al, Coincidence of a high-fluence blazar outburst with a PeV-energy neutrino event, Nature Physics 12 (2016) 807-814 [arXiv:1602.02012]

[8] IceCube Collaboration, Evidence for High-Energy Extraterrestrial Neutrinos at the IceCube Detector, Science 342 (2013) 1242856 [arXiv:1311.5238]

[9] IceCube Collaboration, Multimessenger observations of a flaring blazar coincident with high-energy neutrino IceCube-170922A, Science 361 eaat1378 (2018) [arXiv:1807.08816]

[10] Aartsen et. all, Searches for Time-dependent Neutrino Sources with IceCube Data from 2008 to 2012, ApJ 807 (2015) 46 [arXiv:1503.00598]

[11] The ANTARES Collaboration, Search for muon-neutrino emission from GeV and TeV gamma-ray flaring blazars using five years of data of the ANTARES telescope, J. Cosmology Astropart. Phys. 12 (2015) 014 [arXiv:1506.07354] 
[12] Krauß et. all, Fermi/LAT counterparts of IceCube neutrinos above 100 TeV, A\&A 620 (2018) A174 [arXiv:1810.08482]

[13] Gao et. all, Modelling the coincident observation of a high-energy neutrino and a bright blazar flare, Nature Astronomy 3 (2019) 88-92 [arXiv:1807.04275]

[14] Oikonomou et. all, High-Energy Neutrinos from Blazar Flares and Implications of TXS 0506+056, European Physical Journal Web of Conferences (2019) [arXiv:1903.02006]

[15] Keivani et. all, Astrophysical Multimessenger Observatory Network (AMON): Science, Infrastructure, and Status, in proceedings of ICRC2017, POS ( ICRC2017) 629 (2017).

[16] Reimer et. all, Cascading Constraints from Neutrino Emitting Blazars: The case of TXS 0506+056, arXiv e-prints (2018) [arXiv:1812.05654]

[17] A. Mücke et. al, Monte Carlo simulations of photohadronic processes in astrophysics, Computer Physics Communications 124 (2000) 290-314 [arXiv:astro-ph/9903478]

[18] S. Cutini, Fermi LAT detection of renewed and strong GeV activity from blazar 3C 279, The Astronomer's Telegram 7633 (2015)

[19] F. D'Ammando and M. Orienti, Fermi LAT detection of a GeV flare from the BL Lac object Mrk 421, The Astronomer's Telegram 4261 (2012)

[20] S. Garrappa and S. Buson, Fermi-LAT detection of increasing gamma-ray activity of the blazar BL Lacertae, The Astronomer's Telegram 12718 (2019)

[21] Albert et. all, The Search for Neutrinos from TXS 0506+056 with the ANTARES Telescope, ApJ 863 (2018) L30 [arXiv:1807.04309]

[22] A. Coleiro and D. Dornic, Search for counterpart to IceCube-180908A with ANTARES, The Astronomer's Telegram 12024 (2018)

[23] M. Kreter, Targeting the mystery of extragalactic neutrino sources - A Multi-Messenger Window to the Extreme Universe -, PhD-thesis Würzburg, 2019

[24] IceCube Collaboration, Neutrino emission from the direction of the blazar TXS 0506+056 prior to the IceCube-170922A alert, Science 361 (2018) 147-151 [arXiv:1807.08794]

[25] Perkins et. al., AMEGO: Active Galactic Nuclei, in proceedings of ICRC2017, POS ( ICRC2017) 598 (2017).

[26] Ojha et. al, Neutrinos, Cosmic Rays, and the MeV Band, BAAS 51 (2019) 431 [arXiv:1903.05765] 